\title{
Detection of Hydrogen Peroxide in vitro and in vivo Using Peroxalate Chemiluminescent Micelles
}

\author{
Iljae Lee, ${ }^{\dagger, \mathrm{a}}$ On Hwang, ${ }^{\dagger, \mathrm{a}}$ Donghyuck Yoo, ${ }^{\dagger}$ Gilson Khang, ${ }^{\dagger}$ and Dongwon Lee ${ }^{\dagger, \ddagger, *}$ \\ ${ }^{\dagger}$ Department of BIN Fusion Technology and \\ ¿Department of Polymer·Nano Science and Technology, Jeonju, Chonbuk 561-756, Korea. *E-mail: dlee@jbnu.ac.kr \\ Received March 2, 2011, Accepted May 10, 2011
}

\begin{abstract}
Hydrogen peroxide plays a key role as a second messenger in the normal cellular signaling but its overproduction has been implicated in various life-threatening diseases. Peroxalate chemiluminescence is the light emission from a three component reaction between peroxalate, hydrogen peroxide and fluorophores. It has proven great potential as a methodology to detect hydrogen peroxide in physiological environments because of its excellent sensitivity and specificity to hydrogen peroxide. We developed chemiluminescent micelles composed of amphiphilic polymers, peroxalate and fluorescent dyes to detect hydrogen peroxide at physiological concentrations. In this work, we studied the relationship between the chemiluminescence reactivity and stability of peroxalate by varying the substitutes on the aryl rings of peroxalate. Alkyl substitutes on the aryl ring of peroxalate increased the stability against water hydrolysis, but diminished the reactivity to hydrogen peroxide. Chemiluminescent micelles encapsulating diphenyl peroxalate showed significantly higher chemiluminescence intensity than the counterpart encapsulating dimethylphenyl or dipropylphenyl peroxalate. Diphenyl peroxalate-encapsulated micelles could detect hydrogen peroxide generated from macrophage cells stimulated by lipopolysaccharide (LPS) and image hydrogen peroxide generated during LPS-induced inflammatory responses in a mouse.
\end{abstract}

Key Words : Hydrogen peroxide, Chemiluminescence, Micelles, Peroxalate ester

\section{Introduction}

Reactive oxygen species (ROS) are very small reactive molecules that contain oxygen atoms and peroxides and form as a natural byproduct of the normal cellular metabolism of oxygen. ${ }^{1}$ They include hydrogen peroxide, superoxide, hydroxyl radical, nitric oxide and peroxynitrite. ROS are an essential physiological regulator and serve as an important biological messenger in cell signal transduction cascades. ${ }^{2,3}$ However, the overproduction of ROS leads to oxidative stress, resulting in significant damages to cell structures. Accumulation of oxidative stress damages over time is associated with many life-threatening diseases including cardiovascular diseases, cancer, Alzheimer disease and related neurodegenerative diseases. ${ }^{4}$ In particular, hydrogen peroxide is a major ROS playing a key role as a second messenger in normal cellular signaling. ${ }^{5}$ Recently, numerous publications have suggested that controlled burst of hydrogen peroxide have beneficial roles for cell survival, growth, differentiation and maintenance. ${ }^{6,7}$ However, the overexpression of hydrogen peroxide causes oxidative damages to tissues and organs and has been implicated in inflammatory responses and afore-mentioned life-threatening diseases. ${ }^{8}$ Therefore, hydrogen peroxide has great potential as a diagnostic biomarker of inflammatory responses and there is increasing interest for the development of strategies to detect hydrogen peroxide at physiological concentrations. ${ }^{9,10}$

${ }^{a}$ Both authors contributed equally to this work.
A fluorescent probe, called Amplex red reagents, has been widely used to detect hydrogen peroxide released from human cells. ${ }^{11}$ This probe reacts in a 1:1 stoichiometry with hydrogen peroxide to yield highly fluorescent resorufin in the presence of peroxidase. Amplex red reagents can detect as low as 10 picomoles of hydrogen peroxide. However, its application has drawbacks because of poor selectivity to hydrogen peroxide. Recently, a new methodology has been introduced to detect hydrogen peroxide which exploits peroxalate chemiluminescence reaction. ${ }^{9,12}$ Peroxalate chemiluminescence reaction is a three component reaction between hydrogen peroxide, peroxalate esters and fluorescent dyes. ${ }^{13}$ In principle, peroxalate esters react with hydrogen peroxide to generate dioxetanedione with high energy, which chemically excite fluorescent dyes, leading to the chemiluminescence (Figure 1). Peroxalate chemiluminescence is analogous to ester hydrolysis in which hydrogen peroxide instead of water serves as the substituting nucleophile. ${ }^{13}$

It has great advantages for the detection of hydrogen peroxide due to excellent sensitivity and selectivity to hydrogen peroxide. Bis(2,4,6-trichlorphenyl) oxalate is one of the most widely used peroxalates because of its high reactivity in oxidation by hydrogen peroxide. ${ }^{14,15}$ The electron-withdrawing nature of chlorine on bis(2,4,6-trichlorphenyl) oxalate was known to accelerate addition of hydrogen peroxide. However, the poor stability limits its applications under aqueous conditions because peroxalate linkages react with water to decompose. Previously, Dasari et al. developed chemiluminescent micelles composed of polycaprolactone- 


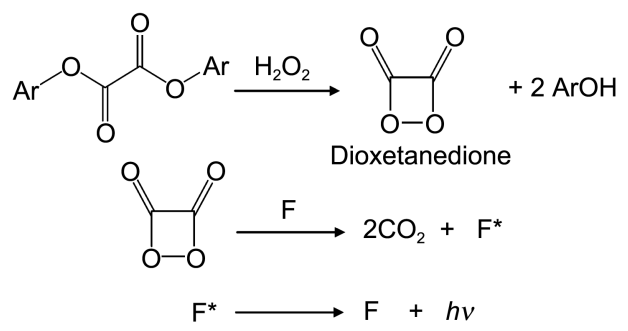

Figure 1. General mechanism of peroxalate chemiluminescence, where $\mathrm{F}$ is the fluorophore and $\mathrm{F}^{*}$ is the singlet excited state of the fluorophore.

co-polyethylene glycol (PCL-PEG) copolymer and diphenyl peroxalate which is more stable than bis(2,4,6-trichlorphenyl) oxalate in water. ${ }^{16}$ Diphenyl peroxalate was encapsulated in the hydrophobic cores and thus protected from water hydrolysis. Diphenyl peroxalate has poor stability against water hydrolysis, but the encapsulation in the hydrophobic core of micelles increased its stability. The micelles could detect hydrogen peroxide at nanomolar concentrations. However, diphenyl peroxalate based chemiluminescent micelles exhibited a half-life of $30 \mathrm{~min}$ in water, which impairs its practical applications for the detection of hydrogen peroxide under aqueous environments.

In this work, we investigated the relationship between the chemiluminescence reactivity and stability by varying the substitutes on the aryl rings of peroxalate. We synthesized the dipropylphenyl peroxalate and dimethylphenyl peroxalate based on the rationale that alkyl groups in the aryl group disfavor the addition of nucleophilic attack of water, increasing the stability of oxalate ester linkages at the expense of reactivity. Chemiluminescent micelles encapsulating various peroxalates could detect hydrogen peroxide with a linear correlation between the hydrogen peroxide concentration and chemiluminescence intensity. In this report, we present the effects of alkyl substitutes on the chemiluminescence reaction, such as emission intensity and duration. We also evaluated the potential of peroxalate chemiluminescent micelles for the detection of hydrogen peroxide using activated macrophages and mice with LPS-induced inflammation.

\section{Materials and Methods}

Preparation of PCL-PEG Copolymers. PCL-PEG copolymers were synthesized as previously reported. ${ }^{17}$ Methoxy poly(ethyleneglycol) (mPEG, $\mathrm{Mn}=2,000)(0.4 \mathrm{mmol})$ and toluene were added into a flask which was dried by heating in a vacuum. The solution was distilled by azeotropic distillation to remove water and then toluene was completely distilled off. Dry dichloromethane (DCM) was added to dried mPEG, followed by the addition of dry caprolactone $(11.2 \mathrm{mmol})$ using a syringe. The polymerization was initiated by the addition of $1.0 \mathrm{M}$ solution of $\mathrm{HCl}$ in diethyl ether $(0.8 \mathrm{mmol})$ at room temperature. After $24 \mathrm{~h}$, the reaction mixture was poured into cold hexane to precipitate polymers. The obtained polymer was redissolved in DCM and filtered off. The polymer solution was concentrated by a rotatory evaporator and dried under vacuum. The chemical structure of PCL-PEG copolymer was determined by ${ }^{1} \mathrm{H}$ NMR: ${ }^{1} \mathrm{H}$ NMR $\left(\mathrm{CDCl}_{3}, 400 \mathrm{MHz}\right): \delta 4.05(\mathrm{t}, 2 \mathrm{H})$, 3.63 (s, 4H), 3.37 (s, 3H), 2.30 (t, Hz, 2H), 1.64 (m, 4H), $1.37(\mathrm{~m}, 2 \mathrm{H})$. The molecular weight was determined by GPC using polystyrene standards.

Synthesis of Dipropylphenyl Peroxalate and Dimethyl Peroxalate. 4-Propyl phenol or 4-methyl phenol $(24 \mathrm{mmol})$ was dissolved in $10 \mathrm{~mL}$ of dry DCM at $0{ }^{\circ} \mathrm{C}$ under nitrogen. Oxalic chloride $(12 \mathrm{mmol})$ was added to the solution dropwise using a syringe and the mixture was stirred for $4 \mathrm{~h}$ at room temperature. The reaction mixture was extracted with DCM, dried on sodium sulfate, filtered and concentrated to get crude dipropylphenyl peroxalates. The crude compound was purified using as silica gel chromatography (hexane/ ethyl acetate $=80: 20$ ). The chemical structure was determined by ${ }^{1} \mathrm{H}$ NMR: ${ }^{1} \mathrm{H}$ NMR $\left(\mathrm{CDCl}_{3}, 400 \mathrm{MHz}\right)$. Dipropylphenyl peroxalate: $\delta 7.0(m, 3 \mathrm{H}), \delta 2.55(\mathrm{~m}, 2 \mathrm{H}), \delta 1.7(d$, $2 \mathrm{H}), \delta 0.9(s, 3 \mathrm{H})$ and methylphenyl peroxalate: $\delta 7.0(\mathrm{~m}$, $3 \mathrm{H}), \delta 2.35(\mathrm{~m}, 3 \mathrm{H})$.

Preparation of Chemiluminescent Micelles. Chemiluminescent micelles were prepared via a solvent displacement method. Rubrene (1 mg), PCL-PEG $(50 \mathrm{mg})$ and peroxalate $(10 \mathrm{mg})$ were dissolved in $1 \mathrm{~mL}$ of acetone, to which $10 \mathrm{~mL}$ of phosphate buffer solution ( $\mathrm{pH}$ 7.4, $0.1 \mathrm{M}$ ) was added. The mixture was vortexed for $10 \mathrm{sec}$ and acetone was evaporated using a rotary evaporator to generate chemiluminescent micelles with a concentration of $2 \mathrm{mg} / \mathrm{mL}$. The particle size of micelles in PBS was measured by dynamic light scattering using a particle analyzer (ELS-8000, Photal Otsuka Electronics, Japan).

Cytotoxicity Assay. The cytotoxicity of micelles was investigated using a 3-(4,5-dimethylthiazol-2-yl)-2,5-diphenyltetrazolium bromide (MTT) reduction assay. RAW 264.7 macrophage cells were seeded at a density of $1 \times 10^{6}$ cells/well in a 24 well plate and incubated for $24 \mathrm{~h}$ to reach $\sim 90 \%$ confluency. Cells were treated with various amounts of micelles $(10 \mu \mathrm{g} / \mathrm{mL}$ to $100 \mu \mathrm{g} / \mathrm{mL})$ and incubated for 1 day. Each well was given $100 \mu \mathrm{L}$ of MTT solution and were incubated for $4 \mathrm{~h}$. Two hundred microlitters of dimethyl sulfoxide (DMSO) was added to cells to dissolve the resulting formazan crystals. After 10 min of incubation, the absorbance at $570 \mathrm{~nm}$ was measured using a microplate reader (Thermolex, Molecular Device Co.). The cell viability was obtained by comparing the absorbance of nanoparticlestreated cells to that of control cells.

Detection of Hydrogen Peroxide. Various concentrations of hydrogen peroxide solutions were prepared in $0.1 \mathrm{M}$ phosphate buffer, $\mathrm{pH}$ 7.4. Hydrogen peroxide solutions were added to each micelle solutions $(2 \mathrm{mg} / \mathrm{mL})$ in a test tube and immediately their chemiluminescence was measured using a luminometer (FB12, Zylux, US) with an acquisition time of $10 \mathrm{~s}$.

Detection of Hydrogen Peroxide Generated in Macrophages. Mouse macrophage RAW 264.7 cells were cultured in DMEM (Dulbecco's Modified Eagle Medium) in a 6-well 
cell culture plate. When cells reached $\sim 90 \%$ confluency, the medium was replaced with Krebs Ringer-buffer. Cells were treated with $1 \mu \mathrm{L}$ or $2 \mu \mathrm{L}$ of LPS $(1 \mathrm{mg} / \mathrm{mL})$ for $1 \mathrm{~h}$ to induce the generation of hydrogen peroxide. Culture medium of $800 \mu \mathrm{L}$ was taken and mixed with $200 \mu \mathrm{L}$ of freshly made chemiluminescent micelles and immediately the chemiluminescence intensity was measured using a luminometer.

Imaging of Hydrogen Peroxide. One milliliter of chemiluminescent micelles was placed in a 6-well cell culture plates. To each well, hydrogen peroxide solutions with various concentrations were added and the plate was gently shaken rapidly. The chemiluminescent emission from the micelles was imaged using an IVIS-200 imaging system (Xenogen, US) for $1 \mathrm{~min}$ acquisition time.

In vivo Chemiluminescence Imaging of Inflamed Mouse Ankle. The ankle of 4 week old mice was injected with $2 \mu \mathrm{L}$ of LPS $(1 \mu \mathrm{g} / \mu \mathrm{L})$ to induce inflammation. Twenty microlitters of micelles $(1 \mu \mathrm{g} / \mu \mathrm{L})$ was injected into the inflamed ankle $4 \mathrm{~h}$ after LPS treatment. Chemiluminescent images of inflamed ankle were made using an IVIS-200 imaging system with 1 min acquisition.

\section{Results and Discussion}

Synthesis of PCL-PEG Copolymers and Alkyl Substituted Phenyl Peroxalate. PCL-PEG copolymers were synthesized by the ring-opening polymerization of caprolactone by terminal alcohol of MPEG as an initiator in the presence of $\mathrm{HCl} \cdot$ ethylene ether. $^{17}$ In this reaction, $\mathrm{HCl}$ serves as an activator, but not as an initiator. This polymerization method has been considered as a potential alternative for simple polymerization of PCL-PEG copolymers with a well defined-structure. The PCL-PEG copolymers were obtained in almost quantitative yield. Its chemical structure was confirmed by NMR (Figure 2). The molecular weight was determined by $\sim 4,500 \mathrm{Da}$ with molecular weigh distribution of $\sim 1.2$.

We developed dimethylphenyl peroxalate and dipropylphenyl peroxalate in which electron-donating alkyl substituents attached to the aryl group increase the stability against water hydrolysis (Figure 3 ). The reaction proceeded in dry DCM under nitrogen to generate the corresponding

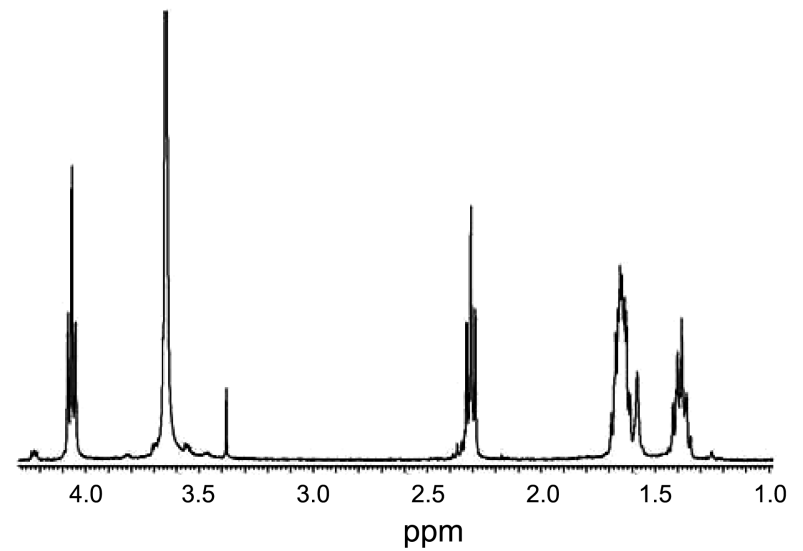

Figure 2. ${ }^{1} \mathrm{H}$ NMR spectrum of PCL-PEG in $\mathrm{CDCl}_{3}$.

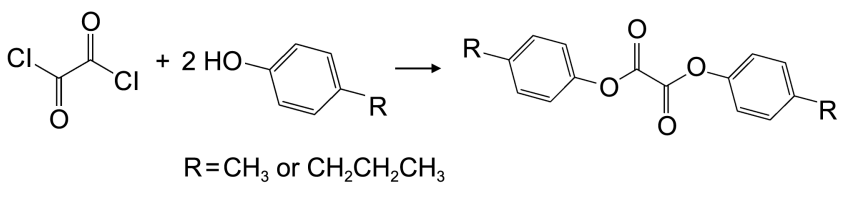

Figure 3. Synthesis of alkyl substituted phenyl peroxalate.

alkyl substituted phenyl peroxalate in $\sim 70 \%$ yield. The products were obtained as pale yellow solids after a silica gel chromatography and drying under high vacuum and their chemical structure was confirmed by ${ }^{1} \mathrm{H}$ NMR.

Development of Chemiluminescent Micelles. A three component peroxalate chemiluminescence has a great potential to detect hydrogen peroxide in vivo and in vitro because of its excellent sensitivity and specificity. ${ }^{9}$ However, the detection of hydrogen peroxide exploiting peroxalate chemiluminescence needs nano-sized structures which can sequester oxalate esters and fluorescent dyes in close proximity and

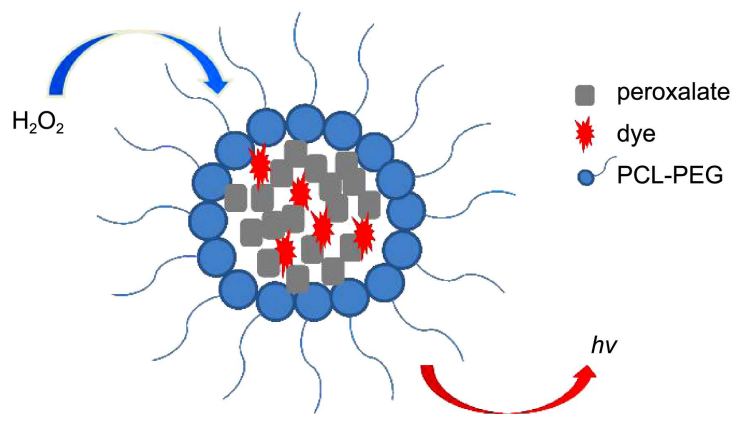

Figure 4. A schematic diagram of chemiluminescent micelles composed of PCL-PEG copolymer, peroxalate and fluorescent dye. The micelles generate light emission instantaneously in response to hydrogen peroxide.
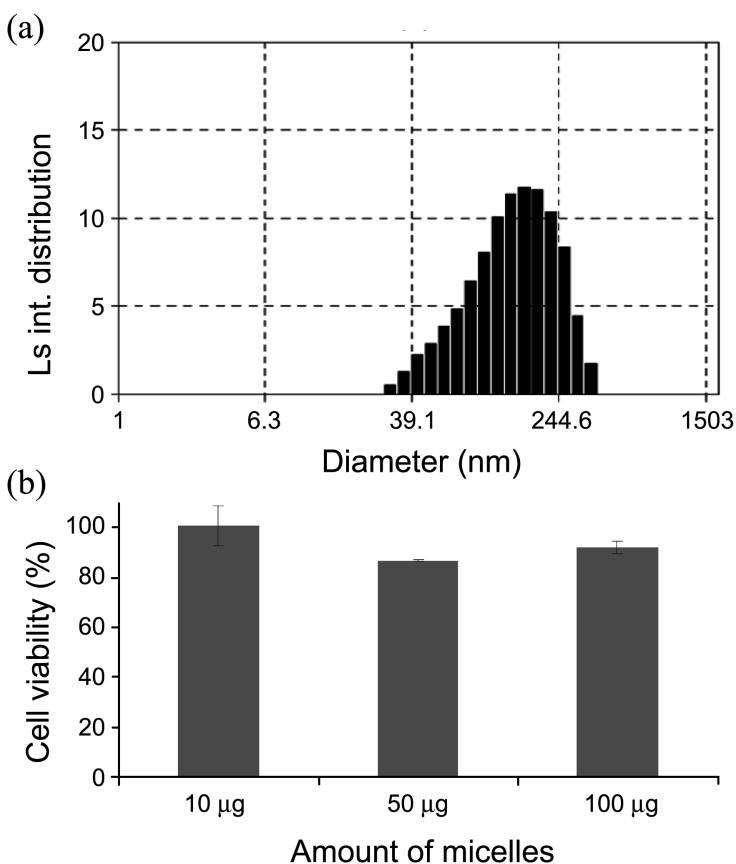

Figure 5. Characterization of chemiluminescent micelles composed of PCL-PEG copolymer, peroxalate and fluorescent dye. (a) size analysis by dynamic light scattering, (b) cytotoxicity assay of micelles. 
allow them to perform peroxalate chemiluminescence instantaneously when exposed to hydrogen peroxide. Polymer micelles are a useful nanostructured material because of their hydrophobic core which can provide environment for sequestering of peroxalate esters and fluorescent dyes in close proximity, as shown in Figure 4. ${ }^{16,18}$

Chemiluminescent micelles were composed of PCL-PEG, peroxalate and fluorescent rubrene. The micelles had a mean size around $\sim 110 \mathrm{~nm}$ and their size was not influenced by the classification of peroxalate (Figure 5). A MTT assay was performed to evaluate the cytotoxicity of chemiluminescent micelles. No or minimal cytotoxicity was observed with micelles upto $100 \mu \mathrm{g}$ of micelles.

Reactivity of Chemiluminescent Micelles to Hydrogen Peroxide. Chemiluminescence of PCL-PEG micelles containing various peroxalates were examined in water by adding hydrogen peroxide or by photoexcitation. Figure 6 shows the emission spectra of chemiluminescent micelles encapsulating rubrene as a fluorophore. Photoexcitation of the micelles at $530 \mathrm{~nm}$ generated light emission at $560 \mathrm{~nm}$ which is a typical wavelength of rubrene. Upon the addition of $33.3 \mu \mathrm{M}$ of hydrogen peroxide, the micelles performed a chemiluminescence reaction to emit light at the same wavelength. The results demonstrate that PCL-PEG micelles sequester peroxalate and rubrene in close proximity in their hydrophobic core to allow them to perform chemiluminescence reaction in response to hydrogen peroxide. We also investigated the effects of alkyl substitutes of peroxalate on the chemiluminescence reaction. The micelles encapsulating diphenyl peroxalate showed a higher chemiluminescent emission intensity than the counterpart of dimethylphenyl peroxalate when exposed to the same concentration of hydrogen peroxide. The lower chemiluminescent intensity of dimethylphenyl peroxalate-encapsulated micelles is attributed to the presence of electron-donating nature of methyl groups which disfavor the oxidation of peroxalate with hydrogen peroxide. .,10,13 $^{9}$

The sensitivity of chemiluminescent micelles to hydrogen peroxide was measured using a luminometer. Chemiluminescent micelles luminesced instantaneously after exposure to hydrogen peroxide. As shown in Figure 7, the micelles

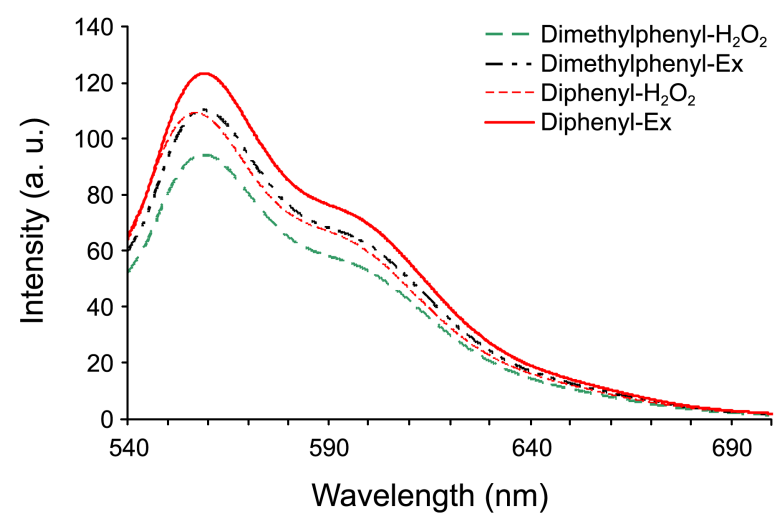

Figure 6. Photoluminescence and chemiluminescence emission spectra of rubrene-encapsulated chemiluminescent micelles.

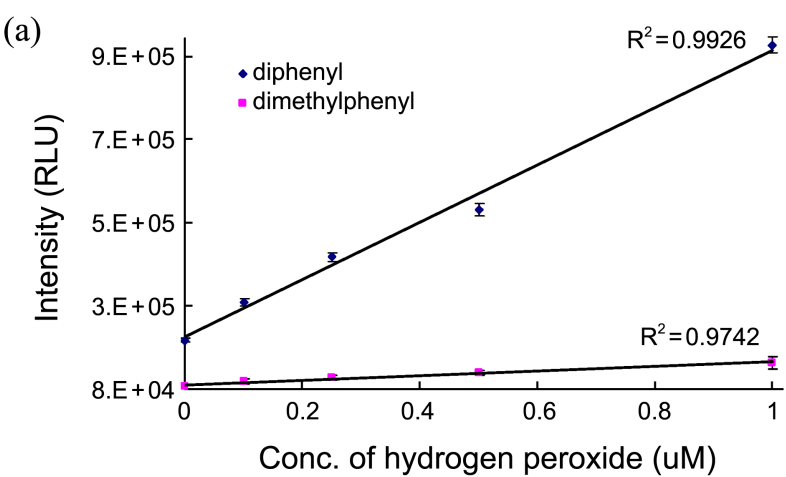

(b)

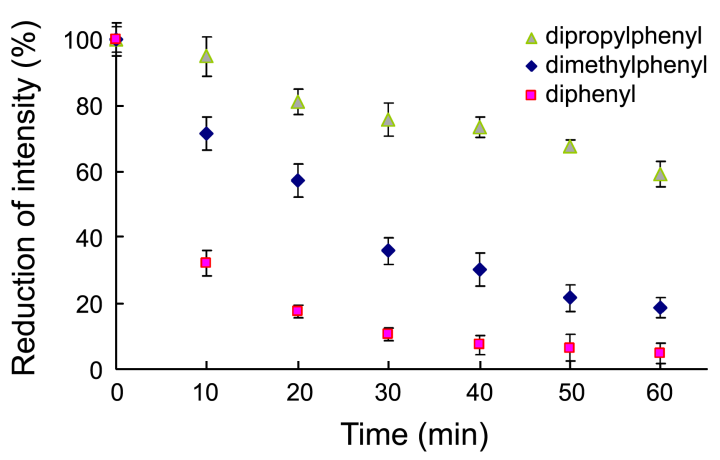

Figure 7. Reactivity of chemiluminescent micelles with hydrogen peroxide. (a) Sensitivity to hydrogen peroxide, (b) Duration of chemiluminescence reaction.

exhibited a linear correlation between chemiluminescent intensity and hydrogen peroxide concentration. However, the alkyl substitute on the phenyl groups influenced the chemiluminescence reactivity. The highest chemiluminescence intensity was observed with diphenyl peroxalate. In the chemiluminescence reaction in the micelles, dimethylphenyl peroxalate generated chemiluminescence with significantly lower intensity than diphenyl peroxalate, but much higher than dipropylphenyl peroxalate (data not shown). The lowest peroxalate chemiluminescence reactivity of dipropylphenyl peroxalate is due to the presence of electron-donating propyl groups which diminish the reactivity to hydrogen peroxideinduced oxidation. ${ }^{13}$

We also investigated the duration of chemiluminescence reaction in micelles by measuring the chemiluminescence intensity during the incubation with hydrogen peroxide (1 $\mu \mathrm{M})$. As shown in Figure 7(b), chemiluminescent micelles encapsulating dipheny peroxalate showed the fastest reduction in chemiluminescence intensity with time, while dipropylphenyl peroxalate encapsulated micelles exhibited the least reduction. The duration of chemiluminescence reaction is highly related to the stability of peroxalate. Although diphenyl peroxalate generated strongest chemiluminescence emission, it showed very short lasting chemiluminescence reactions because of the fast reaction with hydrogen peroxide and water hydrolysis. The results indicate that the alkyl substitutes increase the stability against hydrolysis at the expense of reactivity to hydrogen peroxide.

Detection of Hydrogen Peroxide in Cells. Macrophage cells were stimulated with LPS for $1 \mathrm{~h}$ to produce hydrogen 


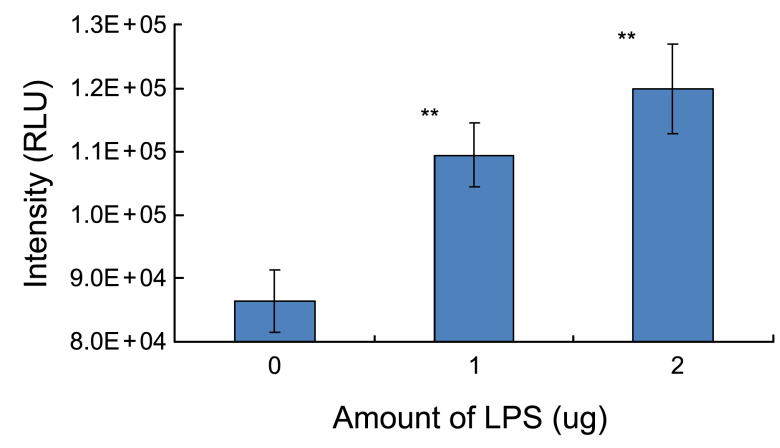

Figure 8. Detection of hydrogen peroxide generated from LPSstimulated macrophages. ${ }^{* *} p<0.01(\mathrm{n}=3)$.

peroxide in the Krebs-Ringer buffer as a culture medium., ${ }^{9,19,20}$ The level of hydrogen peroxide in the Krebs-Ringer buffer was measured by adding chemiluminescent micelles to the buffer and measuring the emission intensity. Figure 8 shows the chemiluminescent intensity of Krebs-Ringer buffer using micelles encapsulating diphenyl peroxalate. Cells treated with LPS produced more hydrogen peroxide, as evidenced by the higher chemiluminescent intensity. The hydrogen peroxide generation was dependent on the amount of LPS given to cells. We also used the micelles encapsulating dimethylphenyl peroxalate. The micelles generated insufficient chemiluminescence intensity to detect hydrogen peroxide generated by activated cells. It is because of the low concentration of hydrogen peroxide generated by stimulated cells and low reactivity of dimethylphenyl peroxalate to hydrogen peroxide.

To demonstrate the potential of chemiluminescent micelles for in vivo imaging, a mouse inflammatory disease model was used. Inflammation was induced in a mouse ankle by intra-articular injection of LPS that causes acute inflammation. ${ }^{9,18}$ Chemiluminescent micelles encapsulating diphenyl peroxalate were injected into the inflamed site at an early stage of inflammation. Figure 9 illustrates a representative in vivo chemiluminescent imaging of hydrogen peroxide in LPS-induced inflammation. Immediately after the

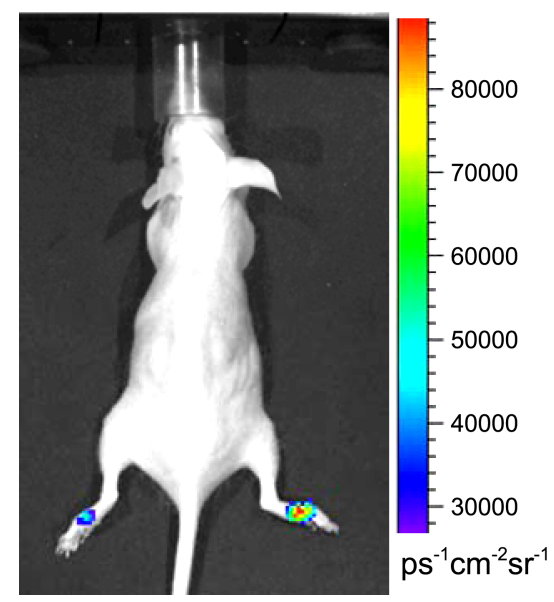

Figure 9. A representative in vivo imaging of hydrogen peroxide in LPS-induced inflammatory responses in a mouse. injection of chemiluminescent micelles, chemiluminescence emission was detected from the LPS-treated inflamed site (right ankle), while LPS-negative control site showed negligible signal (left ankle). The observation demonstrate the potential of chemiluminescent micelles for the detection of hydrogen peroxide-associated inflammatory diseases. ${ }^{18}$

\section{Conclusions}

Chemiluminescent micelles were prepared by self-assembly of amphiphilic PCL-PEG which sequester peroxalate and fluorescent dyes in their hydrophobic core. The addition of hydrogen peroxide generated light emission from the micelles through the three component chemiluminescence reaction, evidenced by the spectra of chemiluminescence emission. The linear correlation was observed between the chemiluminescent intensity and the concentration of hydrogen peroxide. Chemiluminescent micelles composed of diphenyl peroxalate showed a higher emission intensity than those encapsulating alkyl substituted diphenyl peroxalate. However, alkyl substitutes increased the stability against water hydrolysis. Chemiluminescent micelles composed of diphenyl peroxalate could detect hydrogen peroxide generated from the macrophages activated by LPS. In addition, they were able to image hydrogen peroxide generated in LPS-treated inflamed joint of mice. Taken together, the chemiluminescent micelles have great potential to detect hydrogen peroxide in biological environments.

Acknowledgments. This work is supported by a grant of the National Research Foundation of Korea (2009-0063931, 2010-0021903) and World Class University Project (R3120029) by the Ministry of Education, Science and Technology.

\section{References}

1. Azad, N.; Rojanasakul, Y.; Vallyathan, V. Journal of Toxicology and Environmental Health-Part B-Critical Reviews 2008, 11(1), 1.

2. Miller, E. W.; Albers, A. E.; Pralle, A.; Isacoff, E. Y.; Chang, C. J. Journal of the American Chemical Society 2005, 127(47), 16652.

3. Lee, J. Y.; Jang, Y. W.; Kang, H. S.; Moon, H.; Sim, S. S.; Kim, C. J. Archives of Pharmacal Research 2006, 29(10), 849.

4. Chang, M. C. Y.; Pralle, A.; Isacoff, E. Y.; Chang, C. J. Journal of the American Chemical Society 2004, 126(47), 15392.

5. Carter, W. O.; Narayanan, P. K.; Robinson, J. P. Journal of Leukocyte Biology 1994, 55(2), 253.

6. Lang, J. D.; McArdle, P. J.; O'Reilly, P. J.; Matalon, S. Chest 2002, 122(6), 314S.

7. Feder, L. S.; Stelts, D.; Chapman, R. W.; Manfra, D.; Crawley, Y.; Jones, H.; Minnicozzi, M.; Fernandez, X.; Paster, T.; Egan, R. W.; Kreutner, W.; Kung, T. T. American Journal of Respiratory Cell and Molecular Biology 1997, 17(4), 436.

8. Park, H.; Kim, S.; Song, Y.; Seung, K.; Hong, D.; Khang, G.; Lee, D. Biomacromolecules 2010, 11(8), 2103.

9. Lee, D.; Khaja, S.; Velasquez-Castano, J. C.; Dasari, M.; Sun, C.; Petros, J.; Taylor, W. R.; Murthy, N. Nature Materials 2007, 6, 765.

10. Lee, D. W.; Erigala, V. R.; Dasari, M.; Yu, J. H.; Dickson, R. M.; Murthy, N. International Journal of Nanomedicine 2008, 3(4), 
471

11. Soh, N. Analytical and Bioanalytical Chemistry 2006, 386(3), 532.

12. Kamyshny, A.; Magdassi, S. Colloids and Surfaces B-Biointerfaces 1998, $11(5), 249$.

13. Hadd, A. G.; Lehmpuhl, D. W.; Kuck, L. R.; Birks, J. W. Journal of Chemical Education 1999, 76(9), 1237.

14. Motoyoshiya, J.; Sakai, N.; Imai, M.; Yamaguchi, Y.; Koike, R.; Takaguchi, Y.; Aoyama, H. Journal of Organic Chemistry 2002, 67(21), 7314.

15. Maulding, D. R.; Clarke, R. A.; Roberts, B. G.; Rauhut, M. M. The Journal of Organic Chemistry 1968, 33(1), 250.

16. Dasari, M.; Lee, D.; Erigala, V. R.; Murthy, N. Journal of Bio- medical Materials Research Part A 2009, 89A(3), 561.

17. Kim, M. S.; Seo, K. S.; Khang, G.; Cho, S. H.; Lee, H. B. Journal of Biomedical Materials Research Part A 2004, 70A(1), 154.

18. Lim, C. K.; Lee, Y. D.; Na, J.; Oh, J. M.; Her, S.; Kim, K.; Choi, K.; Kim, S.; Kwon, I. C. Advanced Functional Materials 2010, 20(16), 2644.

19. Sredni-Kenigsbuch, D.; Kambayashi, T.; Strassmann, G. Immunology Letters 2000, 71(2), 97.

20. Hikosaka, K.; Koyama, Y.; Motobu, M.; Yamada, M.; Nakamura, K.; Koge, K.; Shimura, K.; Isobe, T.; Tsuji, N.; Kang, C. B.; Hayashidani, H.; Wang, P. C.; Matsumura, M.; Hirota, Y. Bioscience Biotechnology and Biochemistry 2006, 70(12), 2853. 